\title{
Synthesis and Characterization of New 1, 3, 4- Thiadiazoles Substituted with Imidazolidine Moiety
}

\author{
Qasim Mehdi Ismael ${ }^{1}$, FaezAbdul-Hussien Alrammahi ${ }^{1}$, Zeid Hassan Abood $^{3}$ \\ ${ }^{1,2}$ Chemistry Department, College of Education for Women, University of Kufa, Najaf, Iraq; \\ ${ }^{3}$ Chemistry Department, College of Science, University of Kerbala, kerbala, Iraq
}

\begin{abstract}
: 2-amino-5-mercapto-1,3,4-thiadiazolewas introduced in condensation reactions with both terephthaldehyde and 4dimethylaminobenzaldehyde to yield imine derivatives1a and $1 b$ respectively. The resulting imine1awas treated with amino acids $L$ valine and L-cysteine, also the imine $1 b$ wasreacted with amino acids $L$-cysteine, $L$-isoleucine and L-tyrosine to obtain five new imidazolidine derivatives2a-erespectively.2-amino-5-mercapto-1,3,4-thiadiazolewas also converted to the corresponding azoaldehyde derivative3 through coupling reaction of its diazonium salt with 4-hydroxybenzaldehyde dissolved in sodium hydroxide solution. The resulting azoaldehyde 3 was reacted with2-amino-5-mercapto-1,3,4-thiadiazoleto give the corresponding azoimine derivative 4. Treatment of the resulting azoimine 4 with amino acids glycine, L-valine and $L$-cysteine afforded three new imidazolidine derivatives5acrespectively.
\end{abstract}

Keywords: 1,3,4-thiadiazoles, imines, imidazolidines

\section{Introduction}

Thiadiazoles are clear to yellowish liquids which are soluble in alcohol, ether and slightly soluble in water; they are starting material for numerous chemical compounds including sulphur $\operatorname{drugs}^{(1)}$. Thiadiazoles are easily metabolized by biochemical reactions and they are noncarcinogenic in nature $^{(2)}$. Thiadiazoles and their derivatives exhibit wide range of pharmacological activities such as antimicrobial activity ${ }^{(3)}$, antidepressant, cardiotonic $^{(4)}$, antibacterial activity against Klebsiella pneumonia ${ }^{(5)}$, antitubercular $^{(6,7)}$,anticonvulsant $^{(8)}$, antileshmanial analgesic $^{(9)}$, antiinflammtory ${ }^{(10)}$, anticancer $^{(11)}$, phosphodiesterase inhibitors $^{(12)}$ and effect on Tyrosinase enzyme ${ }^{(13)}$.This diversity of biological activity may be due to the presence of $-\mathrm{N}=\mathrm{C}-\mathrm{S}$ moity ${ }^{(14,15)}$. There are four isomers of thiadiazole , among these four isomers 1,3,4-thiadiazole is the most thermally stable; which is only isomer doesn $t$ contain any sulphur- nitrogen bond ${ }^{(16)}$. 1,3,4-thiadiazole relatively stable in aqueous acid solutions but the nucleus can undergo ring cleavage by aqueous base solutions ${ }^{(17)}$.

Imidazolidines are compounds of highly conserved fivemembered ring nitrogen-containing pharmacophores; and thus imidazolidines have attracted attention due to their important roles as building blocks in the synthesis of biologically active compounds ${ }^{(18)}$. Imidazolidine derivatives possess some interesting biological activities such asantiinflammtory, antiviral, antifungal,antihypertensive, antigastrohelcosis and antiasthmatic ${ }^{(19,20)}$. Thus, in this article, we reported here the synthesis of 1,3,4-thiadiazole derivatives containing biologically active imidazolidine moiety, which might have some biological activity.

\section{Experimental}

\subsection{General}

The chemicals used were purchased fromMerck, BDH, sigma Aldrich and $\mathrm{CDH}$ and were used without further purification. Silica TLC plates were used with an aluminum backing $\left(0.2 \mathrm{~mm}, 60 \mathrm{~F}_{254}\right)$. The progress of reactions were monitored by TLC and visualized by development of the TLC plates with iodine vapor. Melting points were determined on an Electro thermal Stuart SMP 30 capillary melting point apparatus. Infrared spectra were recorded on SHIMADZU FTIR-8400S Infrared Spectrophotometer as potassium bromide discs. ${ }^{1} \mathrm{H}$ NMR spectra were collected on NMR spectrometer, Bruker 2009 spectrometer at $400 \mathrm{MHz}$ in DMSO- $\mathrm{d}_{6}$ as solvent and TMS as an internal standard at Kashan University, Iran.Elemental Analysis (CHNS.) was carried out with Perkin Elmer 300A Elemental Analyzer at Kashan University, Iran.Azoaldehyde derivative4was prepared following the method described by Acton ${ }^{(2)}$.

\subsection{Chemical Methods}

\section{Synthesisof5,5'-(((1E,1'E)-1,4-}

phenylenebis(methanylidene))bis(azanylylidene))bis(1,3,4 -thiadiazole-2-thiol) 1a:

Terephthaldehyde( $0.67 \mathrm{~g}, 5 \mathrm{mmol})$ was dissolved in (35 mL) of absolute ethanol, then 2-Amino-5-mercapto-1,3,4thiadiazole $(1.33 \mathrm{~g}, 10 \mathrm{mmol})$ was added. The reaction mixture was refluxed with stirring on a water bath at $65^{\circ} \mathrm{C}$ for $12 \mathrm{~h}$ and monitored by TLC. The mixture was then allowed to cool down to room temperature , the colored precipitate was filtered and recrystallized from ethanol: IR $\left(\mathrm{cm}^{-1}\right): 3194_{\mathrm{br}}$ ( $\mathrm{V} \mathrm{N}-\mathrm{H}$, thione form and $\mathrm{V} \mathrm{C}-\mathrm{H}$, benzene, vib. coupling), 2972and 2885 ( $\mathrm{v} \mathrm{N}-\mathrm{H}$, intramolecularly hydrogen bonded, thione form),

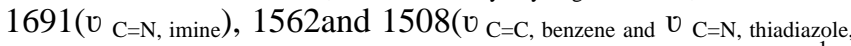
vib. coupling), 1051 ( $\left.\mathrm{V}_{\mathrm{C}=\mathrm{S} \text {, thione form }}\right), 750$ ( $\left.\left.\delta_{\text {o.o.p. C-H, benzene }}\right)\right) ;{ }^{1} \mathrm{H}$ NMR: $\delta=2.5$ (DMSO solvent), $3.4\left(\mathrm{H}_{2} \mathrm{O}\right.$ in DMSO),7.097.96(m, 4H, Ar-H ), 8.10 (s, $2 \mathrm{H}, 2 \times \mathrm{N}-\mathrm{H}$, thione forms), 10.12 (s, $2 \mathrm{H}, 2 \times \mathrm{CH}=\mathrm{N}$, imine).

Synthesis of (Z)-5-((4-(dimethylamino) benzylidene) amino)-(1,3,4-thiadiazole-2-thiol) 1b:

4-Dimethylaminobenzaldehyde(0.745 g, $5 \mathrm{mmol})$ was dissolved in $(30 \mathrm{~mL})$ of absolute ethanol, then 2-Amino-5mercapto-1,3,4-thiadiazole (0.665 g , 5mmol) was added. 


\section{International Journal of Science and Research (IJSR) \\ ISSN (Online): 2319-7064 \\ Index Copernicus Value (2013): 6.14 | Impact Factor (2015): 6.391}

The reaction mixture was refluxed with stirring on a water bath at $65^{\circ} \mathrm{C}$ for $8 \mathrm{~h}$ and monitored by TLC. The mixture was then allowed to cool down to room temperature, the colored precipitate was filtered and recrystallized from ethanol: IR $\left(\mathrm{cm}^{-1}\right): 3090_{\mathrm{br}}(\mathrm{O} \mathrm{N}-\mathrm{H}$, thione form and $\mathrm{V} \mathrm{C}-\mathrm{H}$, benzene, vib. coupling), 2953and 2885 ( $\mathrm{O}$ C-H3 and $\mathrm{V}$ N-H, intramolecularly hydrogen bonded, thione form, vib. coupling), 1597( $\mathrm{V} C=\mathrm{N}$, imine), $1529(\mathrm{~V} \mathrm{C}=\mathrm{C}$, benzene and $\mathrm{V}$ $\mathrm{C}=\mathrm{N}$, thiadiazole, vib. coupling $), 1060$ ( $\mathrm{v} \mathrm{C}=\mathrm{S}$, thione form $), 775$ ( $\delta_{\text {o.o.p. C-H, }}$ benzene); ${ }^{1} \mathrm{H}$ NMR: $\delta=2.49$ (DMSO solvent), 3.02 (s, 6H, $\left.2 \times \mathrm{N}-\mathrm{CH}_{3}\right), 6.62-6.64(\mathrm{~m}, 2 \mathrm{H}, \mathrm{Ar}-\mathrm{H}), 7.59-7.68$ (m, 2H,Ar$\mathrm{H}), 8.20(\mathrm{~s}, 1 \mathrm{H}, \mathrm{N}-\mathrm{H}$, thione form), $9.60(\mathrm{~s}, 1 \mathrm{H}, \mathrm{CH}=\mathrm{N}$, imine).

General procedure for the synthesis of imidazolidine derivatives (2a,2b):

A mixture of bisimine derivative1a $(0.729 \mathrm{~g}, 2 \mathrm{mmol})$ and L-valine or L-cysteine (4mmol) in tetrahydrofuran $(25 \mathrm{~mL})$ was refluxed on a water bath at $70{ }^{\circ} \mathrm{C}$ for about $24 \mathrm{~h}$ and monitored by TLC. The mixture was then allowed to cool down to room temperature, the solvent was evaporated under reduced pressure to give colored solid, driedand recrystallized from ethanol.

2,2'-(1,4-phenylene)bis(5-isopropyl-3-(5-mercapto-1,3,4thiadiazol-2-yl)imidazolidin-4-one)2a:IR $\left(\mathrm{cm}^{-1}\right): 3134_{\mathrm{br}}(v$

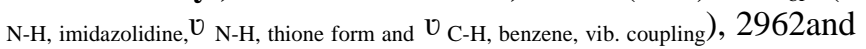
2887 ( $\mathrm{U}$ C-H3 and $\mathrm{V} \mathrm{N}-\mathrm{H}$, intramolecularly hydrogen bonded, thione form, vib.coupling), 1597( $\mathrm{V}_{\mathrm{C}=\mathrm{O}}$, amide, imidazolidine), 1504( $\mathrm{V} \mathrm{C}=\mathrm{C}$, benzene and $\mathrm{V}$ $\mathrm{C}=\mathrm{N}$, thiadiazole, vib. coupling), 1041 ( $\mathrm{C} \mathrm{C}=\mathrm{S}$, thione form $), 773$ and 680 $\left(\delta_{\text {o.o.p. C-H, benzene }}\right) ;{ }^{1} \mathrm{H}$ NMR: $\delta=0.9\left(\mathrm{~d}, 12 \mathrm{H}, 4 \times \mathrm{CH}_{3}\right), 1.8(\mathrm{~m}$, $\left.2 \mathrm{H}, 2 \times \mathrm{CH}\left(\mathrm{CH}_{3}\right)_{2}\right), 2.48$ (DMSO solvent), $3.34\left(\mathrm{H}_{2} \mathrm{O}\right.$ in DMSO),3.64 (s, 2H, 2×O=C-CH-N, imidazolidine), 4.2 (s, $2 \mathrm{H}, 2 \times \mathrm{SH}$ ), 5.26 (s, $2 \mathrm{H}, 2 \times \mathrm{N}-\mathrm{CH}-\mathrm{N}$, imidazolidine), 7.10 7.93(m, $4 \mathrm{H}, \mathrm{Ar}-\mathrm{H}$ ), 8.19 (s, $2 \mathrm{H}, 2 \times \mathrm{N}-\mathrm{H}$, thione forms), 8.30 (s, $2 \mathrm{H}, 2 \times \mathrm{N}-\mathrm{H}$, imidazolidine).Anal. Calcd. for $\mathrm{C}_{22} \mathrm{H}_{26} \mathrm{~N}_{8} \mathrm{O}_{2} \mathrm{~S}_{4}$ : C, 46.96; H, 4.66; N, 19.91; S, 22.79 Found C, 47.28; H, 4.62; N, 20.22; S, 22.47.

2,2'-(1,4-phenylene)bis(3-(5-mercapto-1,3,4-thiadiazol-2yl)-5-(mercaptomethyl) imidazolidin-4-one) 2b:IR (cm $\left.{ }^{1}\right): 3356_{\text {br }}(\mathrm{V}$ N-H, imidazolidine and $\mathrm{N}-\mathrm{H}$, thione form, vib. coupling),3053 and 3032( $v$ C-H, benzene), 2939and 2877 ( $\mathrm{V}$ C-H2 and $\mathrm{V}$ N-H, intramolecularly hydrogen bonded, thione form, vib. coupling),1612( $\mathrm{O} \mathrm{C}=\mathrm{O}$, amide, imidazolidine),

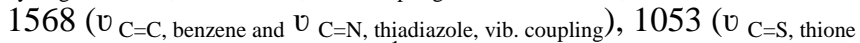
form $), 844$ ( $\left.\delta_{\text {o.o.p. C-H, benzene }}\right) ;{ }^{1} \mathrm{H}$ NMR: $\delta=1.4$ (s, $2 \mathrm{H}, 2 \times \mathrm{CH}_{2}-$ SH), 1.8 (m, 4H, $\left.2 \times \mathrm{CH}_{2}-\mathrm{SH}\right), 2.5$ (DMSO solvent), $3.3\left(\mathrm{H}_{2} \mathrm{O}\right.$ in DMSO), 3.6 (s, $2 \mathrm{H}, 2 \times \mathrm{O}=\mathrm{C}-\mathrm{CH}-\mathrm{N}$, imidazolidine), 4.2 (s, $2 \mathrm{H}, \quad 2 \times \mathrm{SH}$, thiadiazole), 5.26 (s, $2 \mathrm{H}, 2 \times \mathrm{N}-\mathrm{CH}-\mathrm{N}$, imidazolidine), 7.09-7.38(m, 4H, Ar-H ),8.16 (s, 2H, $2 \times \mathrm{N}-$ $\mathrm{H}$, imidazolidine). Anal. Calcd. for $\mathrm{C}_{18} \mathrm{H}_{18} \mathrm{~N}_{8} \mathrm{O}_{2} \mathrm{~S}_{6}$ : C, 37.88; H, 3.18; N, 19.63; S, 33.71 Found C, 38.09; H, 3.26; N, 19.74; S, 34.03.

General procedure for thesynthesis of imidazolidine derivatives (2c,2d,2e):

A mixture of mine derivative1b $(0.525 \mathrm{~g}, 2 \mathrm{mmol})$ and Lcysteine or L-isoleucine or L-tyrosine (2mmol) in tetrahydrofuran $(25 \mathrm{~mL})$ was refluxed on a water bath at 70 ${ }^{\circ} \mathrm{C}$ for about $24 \mathrm{~h}$ and monitored by TLC. The mixture was then allowed to cool down to room temperature, the solvent was removed under reduced pressure to give colored solid, driedand recrystallized from ethanol. 2-(4-(dimethylamino)phenyl)-3-(5-mercapto-1,3,4thiadiazol-2-yl)-5-(mercaptomethyl)imidazolidin-4-

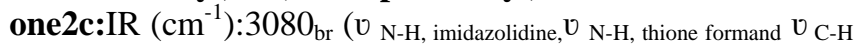
benzene, vib. coupling), 2993and 2887 ( $\mathrm{O}$ C-H3 and $\mathrm{O}$ N-H, intramolecularly hydrogen bonded, thione form, vib. coupling), 2958 and 2825 (U C$\mathrm{H} 2)$, 1593( $\mathrm{V} \mathrm{C}=\mathrm{O}$, amide, imidazolidine), 1533( $\mathrm{V} \mathrm{C}=\mathrm{C}$, benzene and $\mathrm{V} \mathrm{C}=\mathrm{N}$, thiadiazole, vib. coupling $), 1058\left(v_{\mathrm{C}=\mathrm{S} \text {, thione form }}\right), 817$ and $775\left(\delta_{\text {o.o.p. } \mathrm{C}-}\right.$ H, benzene); ${ }^{1} \mathrm{H}$ NMR: $\delta=1.4$ (s, $\left.1 \mathrm{H}, \mathrm{CH}_{2}-\mathrm{SH}\right), 2.31$ (s, $1 \mathrm{H}, \mathrm{CH}_{2^{-}}$ $\mathrm{SH}), 2.48-249$ (DMSO solvent), 3.02 (s, $6 \mathrm{H}, 2 \times \mathrm{N}_{-} \mathrm{CH}_{3}$ ), 3.37 (s, $1 \mathrm{H}, \mathrm{O}=\mathrm{C}-\mathrm{CH}-\mathrm{N}$, imidazolidine),6.7 (s, $1 \mathrm{H}, \mathrm{N}-\mathrm{CH}-\mathrm{N}$, imidazolidine), 7.10(m, 2H, Ar-H), 7.65-7.67 (m, 2H, Ar$\mathrm{H})$, 8.4(s, $1 \mathrm{H}, \mathrm{N}-\mathrm{H}$, thione form) $9.65,(\mathrm{~s}, 1 \mathrm{H}, \mathrm{N}-\mathrm{H}$, imidazolidine); Anal. Calcd. for $\mathrm{C}_{14} \mathrm{H}_{17} \mathrm{~N}_{5} \mathrm{OS}_{3}$ : C, 45.75; $\mathrm{H}$, 4.66; N, 19.05; S, 26.17 Found C, 46.05; H, 4.79; N, 19.22; S, 26.41 .

5-(sec-butyl)-2-(4-(dimethylamino)phenyl)-3-(5mercapto-1,3,4-thiadiazol-2-yl)imidazolidin-4-one 2d:IR $\left(\mathrm{cm}^{-1}\right): 3084_{\mathrm{br}}(\mathrm{V} \mathrm{N}-\mathrm{H}$, imidazolidine, $\mathrm{V} \mathrm{N}-\mathrm{H}$, thione formand $\mathrm{V} \mathrm{C}-\mathrm{H}$, benzene, vib. coupling), 2962and 2889 ( $\mathrm{V}$ C-H3 and $\mathrm{N}-\mathrm{H}$, intramolecularly hydrogen bonded, thione form, vib. coupling),2615( $\mathrm{V}$ S-H,thiol form),1591( $\mathrm{V} \mathrm{C}=\mathrm{O}$, amide,

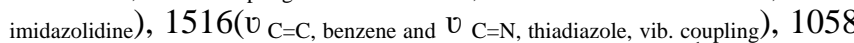
( $0 \mathrm{C}=\mathrm{S}$, thione form $), 775$ and 671 ( $\delta_{\text {o.o.p. C-H, benzene); }}{ }^{1} \mathrm{H}$ NMR: $\delta$ $=0.9\left(\mathrm{~m}, 6 \mathrm{H}, \mathrm{CH}_{3}-\mathrm{CH}_{2}\right.$ andCH $\left.\mathrm{H}_{3}-\mathrm{CH}\right), 1.5\left(\mathrm{~m}, 2 \mathrm{H}, \mathrm{CH}_{2}\right), 1.9$ (m, $1 \mathrm{H}, \mathrm{CH}_{3} \mathrm{CH}-\mathrm{CH}_{2}$ ), 2.5(DMSO solvent),3.02 (s, $6 \mathrm{H}, 2 \times \mathrm{N}$ $\mathrm{CH}_{3}$ ), 3.5 (s, 1H, O=C-CH-N, imidazolidine), 6.7 (s, $1 \mathrm{H}, \mathrm{N}-$ CH-N, imidazolidine), ), 7.10 (m, 2H, Ar-H), 7.54-7.67 (m, $2 \mathrm{H}, \mathrm{Ar}-\mathrm{H}$ ), 8.1 (s, $1 \mathrm{H}, \mathrm{N}-\mathrm{H}$, thione form)9.65, (s, $1 \mathrm{H}, \mathrm{N}-\mathrm{H}$, imidazolidine); Anal. Calcd. for $\mathrm{C}_{17} \mathrm{H}_{23} \mathrm{~N}_{5} \mathrm{OS}_{2}$ : C, 54.09; $\mathrm{H}$, 6.14; N, 18.55; S, 16.99 Found C, 54.43; H, 6.26; N, 18.39; S, 16.71 .

2-(4-(dimethylamino)phenyl)-5-(4-hydroxyphenyl)-3-(5mercapto-1,3,4-thiadiazol-2-yl) imidazolidin-4-one 2e:IR $\left(\mathrm{cm}^{-1}\right): 3446_{\mathrm{br}}(\mathrm{O}$ O-H),3203 ( $\mathrm{N}$ N-H, imidazolidine),3091( $\mathrm{v} \mathrm{N}-\mathrm{H}$, thione form),3018( $\mathrm{U}$ C-H, benzene), 2956and 2867 ( $\mathrm{C}$ C-H3 and $\mathrm{V}$ N-H, intramolecularly hydrogen bonded, thione form, vib. coupling),2600( $\mathrm{U}$ s-H,thiol form), 1589( $\mathrm{O} \mathrm{C}=\mathrm{O}$, amide, imidazolidine), 1531and1514( $\mathrm{V} \mathrm{C}=\mathrm{C}$, benzene and $v_{\mathrm{C}=\mathrm{N}}$, thiadiazole, vib. coupling $), 1080$ ( $\mathrm{C} \mathrm{C}=\mathrm{S}$, thione form $), 777\left(\delta_{\text {o.o.p. C-H, }}\right.$ benzene); ${ }^{1} \mathrm{H}$ NMR: $\delta=2.5$ (DMSO solvent), 3.02 (ss, 6H, $2 \times \mathrm{N}-\mathrm{CH}_{3}$ ), 3.38 (s, $1 \mathrm{H}, \mathrm{O}=\mathrm{C}-\mathrm{CH}-\mathrm{N}$, imidazolidine),6.70(s, $1 \mathrm{H}, \quad \mathrm{N}-\mathrm{CH}-\mathrm{N}$, imidazolidine), 6.88-7.72(m, 8H, Ar$\mathrm{H}), 8.04(\mathrm{~s}, 1 \mathrm{H}, \mathrm{N}-\mathrm{H}$, thione form), 9.63 (s, 1H, O-H),9.65, (s, $1 \mathrm{H}, \mathrm{N}-\mathrm{H}$, imidazolidine). Anal. Calcd. for $\mathrm{C}_{19} \mathrm{H}_{19} \mathrm{~N}_{5} \mathrm{O}_{2} \mathrm{~S}_{2}$ : C, 55.19; H, 4.63; N, 16.94; S, 15.51 Found C, 55.40; H, 4.57; N, 17.29; S, 15.39 .

Synthesis of 4-hydroxy-5-((5-mercapto-1,3,4-thiadiazolyl)diazenyl)benzaldehyde3:was prepared followingthe method described by Acton $^{(21)}$ as dark orange solid, mp 129$131^{\circ} \mathrm{C}$, yield55\%;IR $\left(\mathrm{cm}^{-1}\right): 3284_{\text {br }}(\mathrm{O} \mathrm{O}-\mathrm{H}), 3095$ ( $\mathrm{O}$ N-H, thione

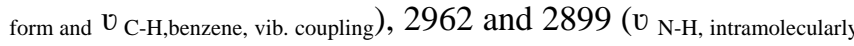
hydrogen bonded, thione form), 2829 and 2700 ( $\mathrm{V}$ C-H, aldehyde), 1627 (

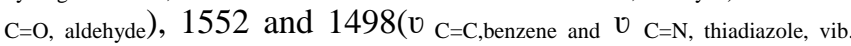
coupling $), 1039\left(v_{\mathrm{C}=\mathrm{S} \text {, thione form }), 837\left(\delta_{\text {o.o.p. }} \mathrm{C}-\mathrm{H} \text {, benzene rings }\right.}\right)$.

Synthesis of 2-((E)-(5-mercapto-1,3,4-thiadiazol-2yl)diazenyl)-4-((E)-((5-mercapto-1,3,4-thiadiazol-2yl)imino)methyl)phenol 4:

Azoaldehyde derivative 3(1.33 g, $5 \mathrm{mmol})$ was dissolved in (30 $\mathrm{mL})$ of absolute ethanol, then 2-Amino-5-mercapto1,3,4-thiadiazole $(0.665 \mathrm{~g}, 5 \mathrm{mmol})$ was added. The reaction 


\section{International Journal of Science and Research (IJSR) \\ ISSN (Online): 2319-7064 \\ Index Copernicus Value (2013): 6.14 | Impact Factor (2015): 6.391}

mixture was refluxed with stirring on a water bath at $65^{\circ} \mathrm{C}$ for $8 \mathrm{~h}$ and monitored by TLC. The mixture was then allowed to cool down to room temperature, the colored precipitate was filtered and recrystallized from ethanol. IR

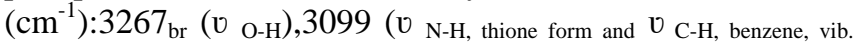
coupling), 2949 and 2802 ( $\mathrm{V} \mathrm{N}-\mathrm{H}$, intramolecularly hydrogen bonded, thione

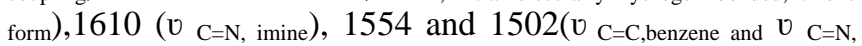
thiadiazole, vib. coupling $), 1061\left(v_{\mathrm{C}=\mathrm{S} \text {, thione form }}\right), 715\left(\delta_{\text {o.o.p. C-H, benzene }}\right.$ rings); ${ }^{1} \mathrm{H}$ NMR: $\delta=2.48$ (DMSO solvent), $3.7\left(\mathrm{H}_{2} \mathrm{O}\right.$ in DMSO)6.8-7.7(m, 3H, Ar-H), 8.7 and 8.8 (ss, $2 \mathrm{H}, 2 \times \mathrm{N}-\mathrm{H}$, thione forms), $9.6(\mathrm{~s}, 1 \mathrm{H}, \mathrm{CH}=\mathrm{N}$, imine),10.60 (s, $1 \mathrm{H}, \mathrm{O}-\mathrm{H})$.

\section{General procedure for the synthesis of imidazolidine derivatives (5a-c):}

A mixture of azomine derivative $4(0.762 \mathrm{~g}, 2 \mathrm{mmol})$ and glycine,L-valine or L-cysteine (2 $\mathrm{mmol})$ in tetrahydrofuran $(25 \mathrm{~mL})$ was refluxed on a water bath at $70{ }^{\circ} \mathrm{C}$ for about $24 \mathrm{~h}$ and monitored by TLC. The mixture was then allowed to cool down to room temperature, the solvent was removed under reduced pressure to give colored solid, driedand recrystallized from ethanol.

(E)-2-(4-hydroxy-3-((5-mercapto-1,3,4-thiadiazol-2yl)diazenyl)phenyl)-3-(5-mercapto-1,3,4-thiadiazol-2-yl) imidazolidin-4-one 5a:IR $\left(\mathrm{cm}^{-1}\right): \quad 3255_{\mathrm{br}}\left(\begin{array}{llll}0 & \mathrm{O}-\mathrm{H}, & \mathrm{V} & \mathrm{N}-\mathrm{H},\end{array}\right.$

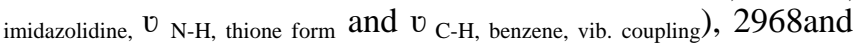
2877 ( $\mathrm{V}$ C-H2, imidazolidine and $\mathrm{V} \mathrm{N}-\mathrm{H}$, intramolecularly hydrogen bonded, thione

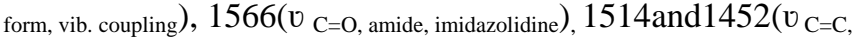

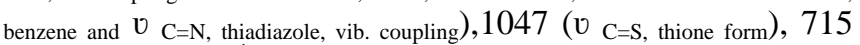
$\left(\delta_{\text {o.o.p. C-H, benzene }}\right) ;{ }^{1} \mathrm{H}$ NMR: $\delta=2.5$ (DMSO solvent), $3.6(\mathrm{~m}$, $2 \mathrm{H}, \quad \mathrm{O}=\mathrm{C}-\mathrm{CH}_{2}-\mathrm{N}$, imidazolidine),6.5(s, $1 \mathrm{H}, \quad \mathrm{N}-\mathrm{CH}-\mathrm{N}$, imidazolidine), 7.10-7.85(m, 3H, Ar-H), 8.18(s, 2H, 2×N-H, thione forms),9.3 (s, $1 \mathrm{H}, \mathrm{N}-\mathrm{H}$, imidazolidine), 13.2 (s, $1 \mathrm{H}$, $\mathrm{O}-\mathrm{H}$ ). Anal. Calcd. for $\mathrm{C}_{13} \mathrm{H}_{10} \mathrm{~N}_{8} \mathrm{O}_{2} \mathrm{~S}_{4}$ : C, 35.61; $\mathrm{H}, 2.30$; N, 25.55; S, 29.25 Found C, 35.78; H, 2.23; N, 25.92; S, 29.19.

\section{2-(4-hydroxy-5-((5-mercapto-1,3,4-thiadiazol-2-} yl)diazenyl)phenyl)-5-isopropyl-3-(5-mercapto-1,3,4thiadiazol-2-yl)imidazolidin-4-one 5b:IR $\left(\mathrm{cm}^{-1}\right): 3188_{\mathrm{br}}(v$

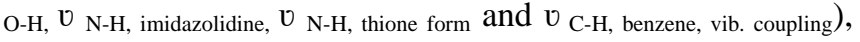
2960and 2879 ( $\mathrm{V} \mathrm{C}-\mathrm{H} 3$, and $\mathrm{V} \mathrm{N}-\mathrm{H}$, intramolecularly hydrogen bonded, thione

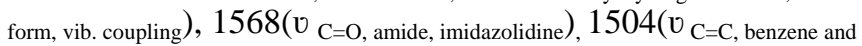
$\left.\mathrm{V}_{\mathrm{C}=\mathrm{N} \text {, thiadiazole, vib. coupling }}\right), 1041$ ( $\mathrm{C} \mathrm{C}=\mathrm{S}$, thione form $), 775\left(\delta_{\text {o.o.p. } \mathrm{C}-\mathrm{H} \text {, }}\right.$ benzene); ${ }^{1} \mathrm{H}$ NMR: $\delta=0.9\left(\mathrm{~m}, 6 \mathrm{H}, \mathrm{CH}\left(\mathrm{CH}_{3}\right)_{2}, 2.48\right.$ (DMSO solvent), $2.86\left(\mathrm{~m}, 1 \mathrm{H}, \mathrm{CH}\left(\mathrm{CH}_{3}\right)_{2}, 3.69(\mathrm{~m}, 1 \mathrm{H}, \mathrm{O}=\mathrm{C}-\mathrm{CH}-\mathrm{N}\right.$, imidazolidine), 5.3 (s, $1 \mathrm{H}, \mathrm{N}-\mathrm{CH}-\mathrm{N}$, imidazolidine), 7.10 7.82(m, 3H, Ar-H), 8.18(s, 2H, $2 \times \mathrm{N}-\mathrm{H}$, thione forms)9.3, (s, $1 \mathrm{H}, \mathrm{N}-\mathrm{H}$, imidazolidine),13.3 (s, $1 \mathrm{H}, \mathrm{O}-\mathrm{H})$. Anal. Calcd. for $\mathrm{C}_{16} \mathrm{H}_{16} \mathrm{~N}_{8} \mathrm{O}_{2} \mathrm{~S}_{4}$ : C, 39.99; H, 3.36; N, 23.31; S, 26.69 Found C, 39.78; H, 3.31; N, 23.10; S, 26.85.

\section{(E)-2-(4-hydroxy-3-((5-mercapto-1,3,4-thiadiazol-2-} yl)diazenyl)phenyl)-3-(5-mercapto-1,3,4-thiadiazol-2-yl)5-(mercaptomethyl)imidazolidin-4-one 5c:IR $\left(\mathrm{cm}^{-1}\right)$ : $3381_{\text {br }}\left(\mathrm{V}_{\mathrm{O}-\mathrm{H}}\right), 3232\left(\mathrm{~V} \mathrm{~N}-\mathrm{H}\right.$, imidazolidine, $\mathrm{V} \mathrm{N}-\mathrm{H}$, thione formand $\mathrm{V}^{\mathrm{C}-\mathrm{H} \text {, benzene, }}$ vib. coupling), 2949and 2877 ( $\mathrm{O}$ C-H2 and $\mathrm{V} \mathrm{N}-\mathrm{H}$, intramolecularly hydrogen bonded, thione form, vib. coupling $), 1566(\mathrm{O} \mathrm{C}=\mathrm{O}$, amide, imidazolidine),

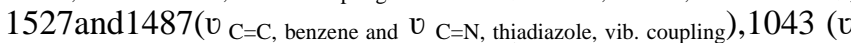
$\mathrm{C}=\mathrm{S}$, thione form $), 845$ ( $\left.\delta_{\text {o.o.p. C-H, benzene); }}\right){ }^{1} \mathrm{H}$ NMR: $\delta=1.8(\mathrm{~s}, 2 \mathrm{H}$, $\mathrm{CH}_{2}$-SH), 2.1 (s, 2H, $\mathbf{C H}_{2}-\mathrm{SH}$ ), 2.49 (DMSO solvent), 3.7 (s, $1 \mathrm{H}, \mathrm{O}=\mathrm{C}-\mathrm{CH}-\mathrm{N}$, imidazolidine),5.4 (s, $1 \mathrm{H}, \quad \mathrm{N}-\mathrm{CH}-\mathrm{N}$, imidazolidine), 7.1-7.8(m, 3H, Ar- $\mathrm{H}), 8.2(\mathrm{~s}, 2 \mathrm{H}, 2 \times \mathrm{N}-\mathrm{H}$, thione forms), $9.7(\mathrm{~s}, 1 \mathrm{H}, \mathrm{N}-\mathrm{H}$, imidazolidine), $13.2(\mathrm{~s}, 1 \mathrm{H}, \mathrm{O}-$ H). Anal. Calcd. for $\mathrm{C}_{14} \mathrm{H}_{12} \mathrm{~N}_{8} \mathrm{O}_{2} \mathrm{~S}_{5}$ : C, 34.70; $\mathrm{H}, 2.50 ; \mathrm{N}$, 23.12; S, 33.08 Found C, 35.04; H, 2.26; N, 23.29; S, 32.91.

\section{Results and Discussion}

\subsection{Chemistry}

The precursors imines 1a and1 b were synthesized by reacting the aromatic aldehydes (terephthaldehyde and 4dimethylaminobenzaldehyde) with 2-amino-5-mercapto1,3,4-thiadiazole in absolute ethanol as indicated in Scheme 1. The synthesized imine 1a was reacted withL-valine and L-cysteine, also the imine derivative 1bwas treatedwithLcysteine, L-isoleucine and L-tyrosine to give the target imidazolidine-1,3,4-thiadiazole derivatives2a-e respectively (Scheme 1). The proposed mechanism for the addition of amino acids to imines was shown in Scheme 2.

The chemical structures of these newlyimidazolidines were confirmedby IR, ${ }^{1} \mathrm{H}$ NMR spectral measurements and (CHNS) elemental analysis and were in good agreement with the proposed structures. The biological activity of the synthesized imidazolidines2a-e will be measured in subsequent study. 


\section{International Journal of Science and Research (IJSR)}

ISSN (Online): 2319-7064

Index Copernicus Value (2013): 6.14 | Impact Factor (2015): 6.391<smiles>Cc1nnc(/N=C/c2ccc(/C=N/c3nnc(S)s3)cc2)s1</smiles><smiles>CN(C)c1ccc(C=O)cc1/C=N\c1nnc(S)s1</smiles>

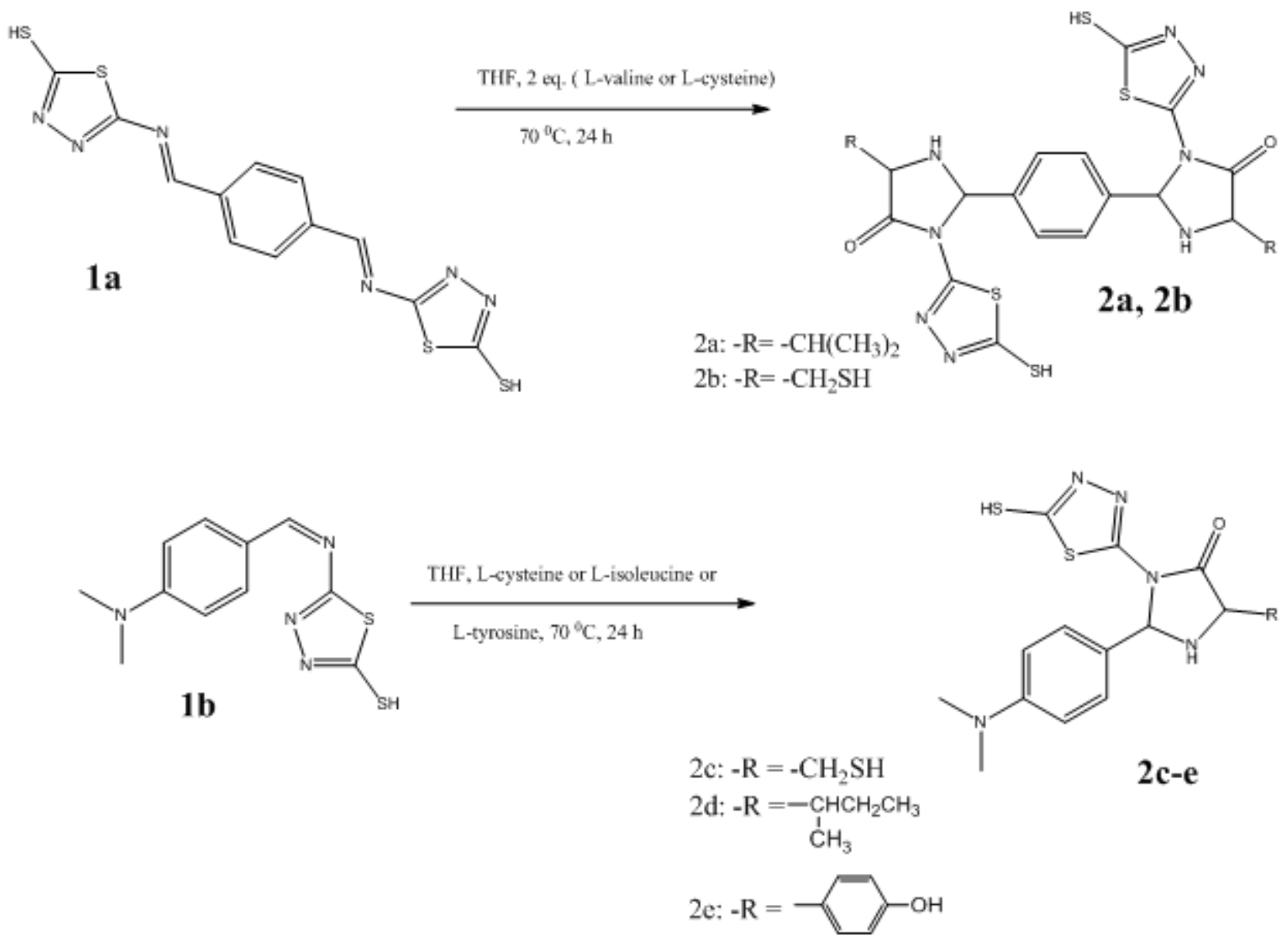

Scheme 1. Synthesis of imidazolidines 2a-e 


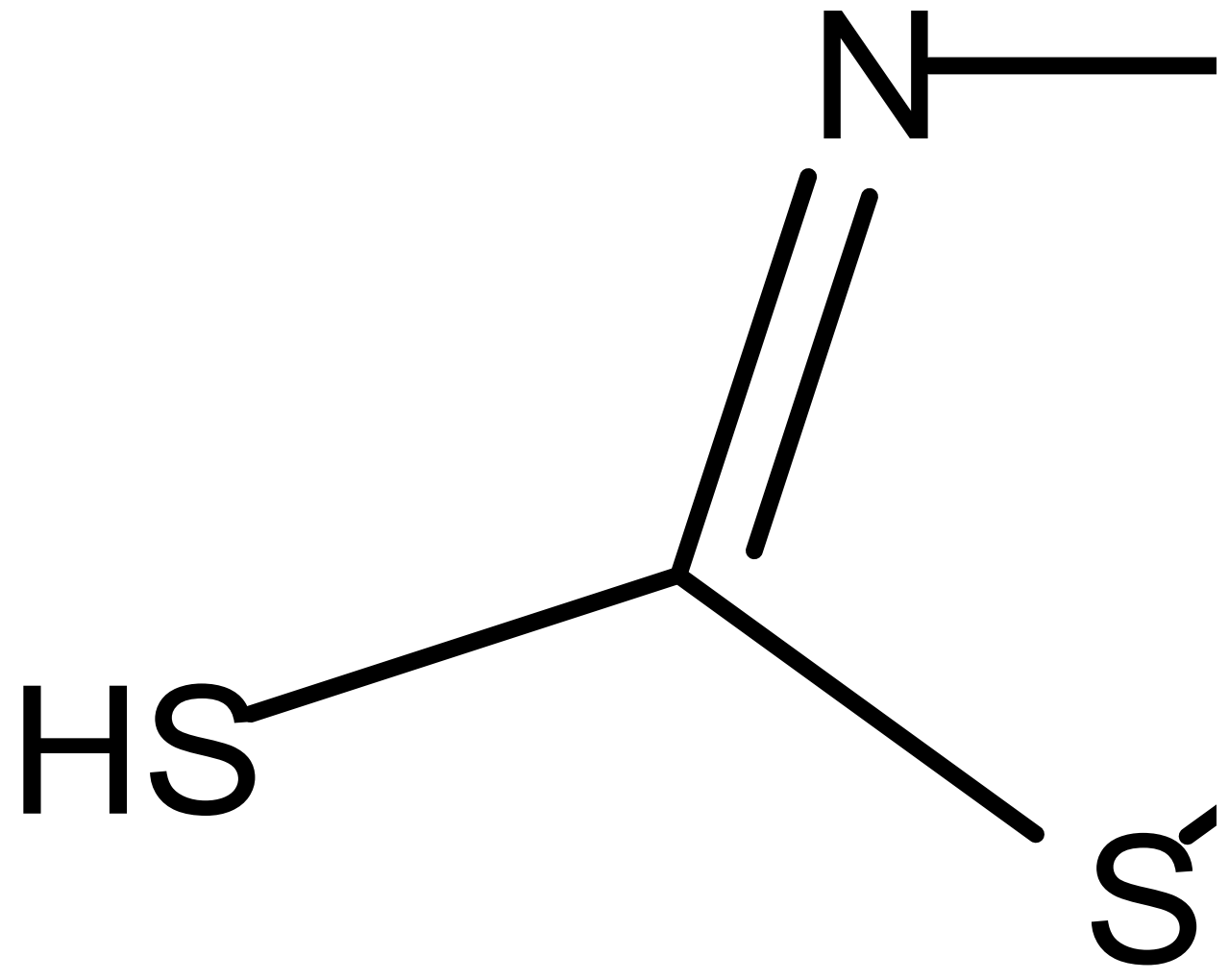

The IR spectra of imines1a and 1bshowed the stretching absorption band of the $(\mathrm{C}=\mathrm{N})$ function at 1691 and $1597 \mathrm{~cm}^{-}$ 1 , respectively, while the absorption bands due to $\left(\mathrm{NH}_{2}\right)$ group at 3336 and $3267 \mathrm{~cm}^{-1}$ have disappeared. The broad absorption band at 3194 and $3090 \mathrm{~cm}^{-1}$ attributed to the $(\mathrm{N}-$ $\mathrm{H})$ str. in thione form, respectively. The stretching band of $(\mathrm{C}=\mathrm{S})$ function in thione form appeared as strong band at 1051 and $1060 \mathrm{~cm}^{-1}$, respectively. The IR spectra of compounds $\mathbf{2 a}$ and $\mathbf{2 b}$ indicated the absence of $(\mathrm{C}=\mathrm{N})$ absorption band and the appearance of $(\mathrm{C}=\mathrm{O}$, amide)str. for imidazolidine ringat1597 and $1612 \mathrm{~cm}^{-1}$, respectively. The stretching absorption band for ( $\mathrm{N}-\mathrm{H}$, imidazolidine) appeared at 3134 and $3356 \mathrm{~cm}^{-1}$, respectively. In the IR spectra of compounds $\mathbf{2 c}, \mathbf{2 d}$ and $\mathbf{2 e}$, the $(\mathrm{C}=\mathrm{O}$, imidazolidine) stretching absorption band was found at 1593, 1591 and $1589 \mathrm{~cm}^{-1}$, respectively, while the absorption band due to $(\mathrm{C}=\mathrm{N})$ has disappeared. The band appearing at 3080, 3084 and $3203 \mathrm{~cm}^{-1}$ was for (N-H, imidazolidine) stretching, respectively. Moreover, the IR spectrum of compound2e showed a characteristic broad band at $3446 \mathrm{~cm}^{-1}$ assigned to the stretching of $(\mathrm{OH})$ function.

The ${ }^{1} \mathrm{H}$ NMR spectra of imines 1a and 1bshowed the $(\mathrm{CH}=\mathrm{N})$ proton as a singlet at $\delta 10.12$ and $9.60 \mathrm{ppm}$ respectively, the $(\mathrm{N}-\mathrm{H})$ proton of thione form appeared as a singlet at 8.10 and $8.20 \mathrm{ppm}$ respectively, the (Ar-H) protons at $\delta 6.62-7.96 \mathrm{ppm}$. Moreover, the spectrum of compound 1b showed the two methyl protons asa singlet at $3.02 \mathrm{ppm}$.

The ${ }^{1} \mathrm{H}$ NMR spectra of imidazolidine compounds 2a and 2b showed the disappearance of the $(\mathrm{CH}=\mathrm{N})$ protonsat 10.12 $\mathrm{ppm}$, the $(\mathrm{O}=\mathrm{C}-\mathrm{CH}-\mathrm{N})$ proton of imidazolidine appeared as a singlet at $3.6 \mathrm{ppm}$,the $(\mathrm{N}-\mathrm{CH}-\mathrm{N})$ proton of imidazolidine as a singlet at5.26ppm, the $(\mathrm{N}-\mathrm{H})$ proton of imidazolidine ring as a singlet at $\delta 8.30$ and $8.16 \mathrm{ppm}$, respectively, The $(\mathrm{Ar}-\mathrm{H})$ protons at $\delta 7.09-7.93 \mathrm{ppm}$, The $(\mathrm{S}-\mathrm{H})$ proton of thiadiazole as a singlet at $4.2 \mathrm{ppm}$. The ${ }^{1} \mathrm{H}$ NMR spectrum of compound2a showed the $(\mathrm{N}-\mathrm{H})$ proton of thione format 8.19 ppm, the fourmethyl protons as doublet at $0.9 \mathrm{ppm}$, while the $\mathbf{C H}\left(\mathrm{CH}_{3}\right)_{2}$ protons appeared asa multiplet at $1.8 \mathrm{ppm}$. The ${ }^{1} \mathrm{H}$ NMRspectrum of compound $2 \mathbf{b}$ showed the two methylene protons at $1.8 \mathrm{ppm}$, the sulfhydryl $\left(\mathrm{CH}_{2}-\mathrm{SH}\right)$ proton as a singlet at $1.4 \mathrm{ppm}$.

The ${ }^{1} \mathrm{H}$ NMR spectra of imidazolidine compounds 2c, 2dand 2e showed the absence of the intense signal due to the $(\mathrm{CH}=\mathrm{N})$ proton at $9.60 \mathrm{ppm}$,the peak for the two methyl $\mathrm{N}\left(\mathrm{CH}_{3}\right)_{2}$ protons appeared at $3.02 \mathrm{ppm}$, the $(\mathrm{O}=\mathrm{C}-\mathrm{CH}-\mathrm{N})$ proton of imidazolidine appeared as a singlet at 3.37, 3.5 and $3.38 \mathrm{ppm}$ respectively, the $(\mathrm{N}-\mathrm{CH}-\mathrm{N})$ proton of imidazolidine as a singlet at $6.7 \mathrm{ppm}$, the $(\mathrm{N}-\mathrm{H})$ proton of imidazolidine ring as a singlet at $\delta 9.65 \mathrm{ppm}$, the singlet at 8.4, 8.1 and 8.04 attributed to the $(\mathrm{N}-\mathrm{H})$ proton in thione form respectively, the $(\mathrm{Ar}-\mathrm{H})$ protons at $\delta$ 6.88-7.72 ppm.The ${ }^{1} \mathrm{H}$ NMR spectrum of compounds2cshowedthe $\left(\mathrm{CH}_{2}-\mathrm{SH}\right)$ proton at $1.4 \mathrm{ppm}$, themethylene protons at 2.31 ppm. The ${ }^{1} \mathrm{H}$ NMR spectrum of compound2d showedthe two methylprotons, methylene protons and methine proton in $\left(\mathrm{CH}_{3}-\mathrm{CH}_{2}-\mathrm{CH}-\mathrm{CH}_{3}\right)$ as multiplets at $0.9,1.5$ and $1.9 \mathrm{ppm}$, respectively. The ${ }^{1} \mathrm{H}$ NMR spectrum of compound2e showedthe $(\mathrm{O}-\mathrm{H})$ proton as a broad signal at $9.63 \mathrm{ppm}$.

Theazoaldehyde derivative3was synthesized by reacting the diazonium salt of2-amino-5-mercapto-1,3,4-thiadiazolewith alkaline solution of 4-hydroxybenzaldehyde using the method described by acton ${ }^{(21)}$ as shown in Scheme 3.The resulting azoaldehyde 3wascondensed with2-amino-5mercapto-1,3,4-thiadiazole in absolute ethanol to give 


\section{International Journal of Science and Research (IJSR) \\ ISSN (Online): 2319-7064 \\ Index Copernicus Value (2013): 6.14 | Impact Factor (2015): 6.391}

azoimine derivative $\mathbf{4}$. The resulting imine4was allowed to react withglycine, L-valine and L-cysteine leading to the formation of imidazolidine-1,3,4-thiadiazole derivatives 5ac respectively (Scheme 3).

The structures of the target compounds synthesized were provenbyIR, ${ }^{1} \mathrm{H}$ NMR spectral measurements and (CHNS) elemental analysis and were in good agreement with the proposed structures. The biological activity of the synthesized imidazolidines $\mathbf{5 a - c}$ will be measured in subsequent study.
The IR spectrum of azoaldehde derivative $\mathbf{3}$ indicated the absence of the doublet band at 3336and $3267 \mathrm{~cm}^{-1}$ for ($\mathrm{NH}_{2}$ )str. and appearance of the following characteristic bands: the broad band at $3284 \mathrm{~cm}^{-1}$ assigned to $(\mathrm{O}-\mathrm{H})$ str., the absorption band at $1627 \mathrm{~cm}^{-1}$ belong to aldehydic $(\mathrm{C}=\mathrm{O})$ str., the stretching absorption bands assigned to $(\mathrm{N}-\mathrm{H})$ and $(\mathrm{C}=\mathrm{S})$ in thione form appeared at 3095 and $1039 \mathrm{~cm}^{-1}$, respectively. The IR spectrum of azoimine derivative $\mathbf{4}$ showed the disappearance of the absorption band at $1627 \mathrm{~cm}^{-1}$ for aldehydic $(\mathrm{C}=\mathrm{O})$ str., also disappearing the doublet band for $\left(-\mathrm{NH}_{2}\right)$ str. in2-amino-5-mercapto-1,3,4-thiadiazole at (3336, 3267) $\mathrm{cm}^{-1}$, while the absorption band attributed to $(\mathrm{C}=\mathrm{N})$ str.appeared at1610 $\mathrm{cm}^{-1}$. In the IR spectra of imidazolidine compounds $\mathbf{5 a}, \mathbf{5 b}$ and $\mathbf{5 c}$, the stretching absorption band due to ( $\mathrm{C}=\mathrm{O}$, imidazolidine) was found at 1566, 1568 and $1566 \mathrm{~cm}^{-1}$, respectively, while the absorption band due to $(\mathrm{C}=\mathrm{N})$ at $1610 \mathrm{~cm}^{-1}$ has disappeared.The band appearing at 3080, 3084 and $3203 \mathrm{~cm}^{-1}$ was for $(\mathrm{N}-\mathrm{H}$, imidazolidine) stretching, respectively.

The ${ }^{1} \mathrm{H}$ NMR spectrum of azoimine compound $\mathbf{4}$ showed the $(\mathrm{O}-\mathrm{H})$ proton as a broad peak at $10.60 \mathrm{ppm}$, the $(\mathrm{CH}=\mathrm{N})$ proton as a singlet at $\delta 9.6 \mathrm{ppm}$, the two $(\mathrm{N}-\mathrm{H})$ protons of thione forms appeared as a singlets at 8.7 and $8.8 \mathrm{ppm}$, the $(\mathrm{Ar}-\mathrm{H})$ protons at $\delta 6.8-7.7 \mathrm{ppm}$.
The ${ }^{1} \mathrm{H}$ NMR spectra of imidazolidine compounds $\mathbf{5 a}$, $\mathbf{5} \mathbf{b}$ and 2c showed the disappearance of the $(\mathrm{CH}=\mathrm{N})$ proton at $\delta 9.6$ ppm,the $\left(\mathrm{O}=\mathrm{C}-\mathrm{CH}_{2}-\mathrm{N}\right)$ protons of imidazolidine in compound 5a appeared at $3.6 \mathrm{ppm}$, the $(\mathrm{O}=\mathrm{C}-\mathrm{CH}-\mathrm{N})$ proton of imidazolidine in compounds $5 \mathbf{b}$ and $5 \mathbf{c a t} 3.7 \mathrm{ppm}$, the $(\mathrm{N}$ $\mathrm{CH}-\mathrm{N}$ ) proton of imidazolidine as a singlet at $6.5,5.3$ and $5.4 \mathrm{ppm}$ respectively, the $(\mathrm{N}-\mathrm{H})$ proton of imidazolidine ring as a singlet at $\delta 9.35,9.35$ and $9.7 \mathrm{ppm}$ respectively, the singlet at 8.18-8.2 attributed to the $(\mathrm{N}-\mathrm{H})$ proton in thione form, the signal of $(\mathrm{O}-\mathrm{H})$ proton appearedas a singlet at 13.2-13.3 ppm, the (Ar-H) protons recorded at $\delta$ 7.10-7.85 ppm; additionally the ${ }^{1} \mathrm{H}$ NMR spectrum of compound $\mathbf{5 b}$ showed the two methyl protons as a multiplet at $0.9 \mathrm{ppm}$, while the $\mathrm{CH}\left(\mathrm{CH}_{3}\right)_{2}$ proton at $2.86 \mathrm{ppm}$. Also, the ${ }^{1} \mathrm{H}$ NMR spectrum of compound5cshowedthe $\left(\mathrm{CH}_{2}-\mathrm{SH}\right)$ proton as a singlet at $1.8 \mathrm{ppm}$, themethylene protons as a singlet at 2.1 ppm. 


\section{International Journal of Science and Research (IJSR) \\ ISSN (Online): 2319-7064}

Index Copernicus Value (2013): 6.14 | Impact Factor (2015): 6.391

Table 1: Physical Properties of the Synthesized Compounds

\begin{tabular}{|c|c|c|c|c|}
\hline Product & $\begin{array}{l}\text { Physical } \\
\text { state }\end{array}$ & $\mathrm{R}_{\mathrm{f}}$ (developer) & m.p. $\left({ }^{\circ} \mathrm{C}\right)$ & $\begin{array}{l}\text { Yield } \\
(\%)\end{array}$ \\
\hline 1a & Yellow solid & 0.64 (Toluene/ EtOH, 7:3) & $186-188$ & 79 \\
\hline $1 \mathrm{~b}$ & Orange solid & 0.77 (Toluene/ EtOH, 7:3) & $174-176$ & 82 \\
\hline $2 \mathbf{a}$ & $\begin{array}{c}\text { Dark orange } \\
\text { solid }\end{array}$ & 0.74(Toluene/ EtOH, 7:3) & $115-117$ & 71 \\
\hline $2 \mathbf{b}$ & $\begin{array}{c}\text { Dark orange } \\
\text { solid }\end{array}$ & 0.80(Toluene/ EtOH, 7:3) & $108-110$ & 68 \\
\hline 2c & $\begin{array}{c}\text { Light brown } \\
\text { solid }\end{array}$ & 0.82(Toluene/ EtOH, 7:3) & $151-153$ & 83 \\
\hline 2d & $\begin{array}{c}\text { Light orange } \\
\text { solid }\end{array}$ & 0.76(Toluene/ EtOH, 7:3) & $159-161$ & 75 \\
\hline $2 e$ & Orange solid & 0.74(Toluene/ EtOH, 7:3) & $165-167$ & 78 \\
\hline 3 & $\begin{array}{c}\text { Dark orange } \\
\text { solid }\end{array}$ & 0.49(Toluene/ EtOH, 7:3) & 129-131 & 55 \\
\hline 4 & Orange solid & 0.73(Toluene/ EtOH, 7:3) & $178-180$ & 71 \\
\hline $5 a$ & Brown solid & 0.75(Toluene/ EtOH, 7:3) & $111-113$ & 74 \\
\hline $5 \mathbf{b}$ & Brown solid & 0.68(Toluene/ EtOH, 7:3) & 117-119 & 73 \\
\hline $5 c$ & Brownsolid & 0.77(Toluene/ EtOH, 7:3) & $114-116$ & 67 \\
\hline
\end{tabular}

\section{Acknowledgements}

We are highly thankful to staff of the central laboratory, University of Kashan, Iran for their significant assistance in ${ }^{1} \mathrm{H}$ NMR and Elemental Analysis measurements of the target compounds.

\section{References}

[1] S. Jalhan, A. Jidal, A. Gupta and Hemraj, Asian journal of pharmaceutical and clinical Reseach, 5, 199(2012).

[2] M.M. Raj, H.V. Patel, L.M. Raj and N.K. Patel, IJPCBS, 3, 814(2013).

[3] S. Adhikari, S.B. Bari, A. Samanta, Journal of Applied Chemical Research, 8, 31(2014).

[4] Society of Ecological Chemistry and Engineering, Influence of 1,3,4-thiadiazole derivatives on the biological activity of the selected environmental bacteria, Opole University, Vol.18,No.12, pp. 16911692 (2011).

[5] N. Aggarwal, R. Kumar, P. Dureja and J.M. Khurana, Chem Bio Drug Des, 79, 384 (2012).

[6] M. Amir, A. Kumar, I. Ali and S.A. khan, Indian J. Chem., 48B, 1288(2009).

[7] S.H. Joshi and M.K. ThaKer,Indian J. Chem., 44B, 410(2005).

[8] B. Ahmed andM.d. Yusuf, Indian J. Chem., 49B, 241 (2010).

[9] D.E. Abdel Rahman and K.O. Mohamed, Der Pharma Chemica, 6, 323(2014).

[10] F. A. Hassan, IJRPC, 2, 58(2012).

[11] A.S.Mayhoub, L. Marler, T.P. Kondratyuk, E.J. Park, J.M. Pezzuto and M. Cushman, Bioorg. Med. Chem., 20,2427(2012).

[12] F. Vergne, P. Bernardelli, E. Lorthiois, N. Pham, E. Proust, C. Oliveira, A. Mafroud and F. Royer,Bioorg. Med. Chem. Lett., 14, 4607(2004).

[13] M.M. El-Sadek, S.Y. Hassan, H.E. Abdelwahab and G.A. Yacout, Molecules, 17, 8378(2012).

[14] E. Oruc, S. Rollas, F. Kandemirli, N. Shavets and A. Dimoglo, J. Med. Chem.,47, 6760(2004).
[15] S. Jaiswal and S. Sigh, International Journal of Engineering Research and General Science, 2, 167 (2014).

[16] S. Alrammahi and F. A. Alrammahi, International Journal of Advanced Multidisciplinary Research, 1, 38 (2014).

[17] Y. Hu, C.Y. Li, X.M. Wang, Y.H. Yang and H.L. Zhu, ACS Publications, 27, A(2013).

[18] W. Beebe, E. F. Dohmeier, D. C. Seaman, C. A. Castro and G. Moura-Letts, $6^{\text {th }}$ Annual Undergraduate Research Symposium, p. 19 (2015)

[19] M.J. Aranjo, J. Bom, R. Capla, C. Casimiro, P. Chambel, P. Gomes, J. Hey, F. Lopes, J. Morais, R. Moreire, E.D. Oliveira, V.D. Rosario and N. Vale, J. Med. Chem., 48, 888 (2005).

[20] M. Wroblewska, J. Kasprzyk, F. Saczewski, A. Kronicka, K. Boblewski, A. Lehmann and A. Rybczyrska, Pharmacological Report, 1025 (2013).

[21] Acton, Q. A., Azo Compounds: Advances in Research and Application, Scholarly Paper Edition, Atlanta, p.42 (2011). 